\author{
Susanne Polywka \\ Heinz-Hubert Feucht \\ Matthias Schröter \\ Rainer Laufs
}

\section{Diagnostik der Hepatitis-C-Virusinfektion: Die Bedeutung von Bestätigungstesten*}

\author{
Necessity of Confirmation Assays in Diagnosis of Infections with the \\ Hepatitis C Virus
}

\section{Zusammenfassung}

Zur Evaluierung der Sensitivität und Spezifität eines kommerziell erhältlichen Tests der dritten Generation zum Nachweis von Antikörpern gegen das Hepatitis-C-Virus (HCV) untersuchten wir Seren von 1000 Patienten mit klinischem Verdacht auf eine HCV-Infektion mit einem Mikropartikel-Enzymimmunoassay (MEIA), einem in unserem Institut entwickelten Bestätigungstest (UKE SIA), sowie der PCR. Vierundvierzig Patienten waren im MEIA negativ, 6 waren im Grauzonenbereich, und bei 950 Patienten war der MEIA reaktiv. Zwei HCV-infizierte Patienten mit wiederholt positivem Ergebnis in der PCR wurden vom Antikörpersuchtest nicht detektiert. Die Häufigkeit, mit der das MEIA-Ergebnis bestätigt werden konnte, hing deutlich von der Höhe der Reaktivität im Antikörpersuchtest ab: lag der S/COWert zwischen 1 und 10, konnten nur 14,3\% der Reaktivitäten als spezifisch bestätigt werden, während 99,6\% der Patienten mit S/CO-Werten $>40$ spezifisch reaktiv waren. Um die Diagnose einer HCV-Infektion verlässlich stellen zu können, sollten alle erstmals mit einem Suchtest gefundenen Reaktivitäten mindestens einmal mit einem Bestätigungstest nachuntersucht werden, der am besten Antigene enthalten sollte, die sich von denen des Suchtests unterscheiden.

\section{Schliuisselwörter}

Hepatitis C · Bestätigungstest · Spezifität · PCR

\section{Abstract}

To evaluate sensitivity and specificity of a commercially available third-generation assay for the detection of antibodies to the hepatitis $\mathrm{C}$ virus (HCV) in a large number of patients with suspected hepatitis $C$ we tested 1000 sera with a microparticle enzyme immuno assay (MEIA), an in-house confirmatory assay (UKE SIA), and by PCR. Forty-four patients were negative in the MEIA, 6 had borderline reactivities, and 950 were reactive. Two HCV-infected patients were missed by the MEIA as shown by a positive PCR result. The rate of confirmed MEIA reactivities clearly depended on the strength of the reactivity. In sera with low S/CO-values between 1 and 10, only $14.3 \%$ were shown to be specifically reactive, while in those exceeding an S/CO-value of $40,99.6 \%$ were proven to be HCV-infected. Therefore, positive reactivities measured by a screening assay should always be confirmed at least once with a confirmatory assay which should contain antigens different from those used in the screening assay.

Key words

Hepatitis C · Confirmatory Assay $\cdot$ Specificity $\cdot$ PCR 
Das Hepatitis-C-Virus (HCV), der wichtigste Erreger der Hepatitis Non-A, Non-B, wurde 1989 durch molekularbiologische Methoden entdeckt [1]. Kurze Zeit später wurden die ersten, auf rekombinanten Proteinen basierenden Tests zum Nachweis HCVspezifischer Antikörper erhältlich [2]. Die Enzymimmunoassays (EIA) der 1. Generation enthielten ein an eine Superoxiddismutase gebundenes Antigen der Nichtstrukturregion (NS) 4, das als c100- 3 bezeichnet wurde. Diese Tests waren weder ausreichend sensitiv noch spezifisch, und so wurden bei den EIAs und Bestätigungstests (rekombinanter Immunoblotassay [RIBA]) der 2. Generation weitere, synthetisch hergestellte Antigene eingeführt. Diese zusätzlichen Antigene stammten aus dem NS3-Protein (c33c, c200) und aus der strukturellen Core-Region (c22-3) [3]. Mittlerweile sind Tests der 3. Generation erhältlich, die ein weiteres nichtstrukturelles Protein (NS5) enthalten [4].

In einer früheren Arbeit beschrieben wir bereits die hohe Rate falsch positiver Reaktivitäten in einem kommerziell erhältlichen EIA der 2. Generation, verglichen mit einem in unserem Institut entwickelten Bestätigungstest (UKE SIA) [5]. Damals waren 13\% aller Seren, die im HCV-EIA reaktiv waren, im Bestätigungstest negativ, und weitere $10 \%$ waren fraglich reaktiv. Seitdem wurde der EIA der 2. Generation durch einen anderen kommerziell erhältlichen, voll automatisierten Mikropartikel-Enzymimmunoassay (MEIA) der 3. Generation ersetzt. Zur Beurteilung, ob der Einschluss des NS5-Proteins die Sensitivität und Spezifität des EIA erhöht hat, verglichen wir in der vorliegenden Studie die MEIA-Reaktivität mit unserem Bestätigungstest und der Polymerase-Kettenreaktion (PCR).

\section{Material und Methoden}

Patienten. Eingeschlossen in die Studie wurden Seren von 1000 Patienten mit Verdacht auf eine HCV-Infektion, die sowohl im MEIA als auch im Bestätigungstest auf HCV-Antikörper untersucht wurden, und bei denen eine Bestimmung der HCV-RNA mittels PCR erfolgte. Das Alter der Patienten lag zwischen 3 und 89 Jahren (Median: 38 Jahre). Von 436 (43,6\%) Patienten waren Risikofaktoren für eine HCV-Infektion bekannt; davon waren $281(64,5 \%)$ intravenös drogenabhängig, $60(13,8 \%)$ hatten anamnestisch Bluttransfusionen erhalten, 39 (8,9\%) waren im medizinischen Bereich tätig, $27(6,2 \%)$ waren chronisch dialysepflichtig, 23 (5,3\%) waren Hämophiliepatienten, 4 (0,9\%) hatten familiäre Kontakte zu HCV-Infizierten und $2(0,5 \%)$ hatten Immunglobulinpräparationen erhalten. Von 802 Patienten waren weitere Serumproben vorhanden; bei diskrepanten Ergebnissen in MEIA und Bestätigungstest und/oder PCR wurden die bei anderer Gelegenheit ermittelten Testergebnisse herangezogen, um eine endgültige Klassifizierung des Patienten in infiziert oder nicht infiziert zu ermöglichen.

Antikörpersuchtest. Als Screeningtest wurde der $\mathrm{AxSYM}^{\circledR} \mathrm{HCV}$ Version 3.0 Microparticle Enzyme Immunoassay (Abbott GmbH Diagnostika, Wiesbaden-Delkenheim) benutzt. Dieser Test enthält 4 rekombinante Proteine: $\mathrm{HCr}$ 42, ein in Escherichia coli (E. coli) exprimiertes Fusionsprotein, das Teile der Core-Region und der NS3-Region enthält; c200, ein in Saccharomyces cerevisiae (S. cerevisiae) exprimiertes und an eine Superoxiddismutase (SOD) gekoppeltes Antigen aus Teilen der NS3- und NS4-Region; c100-3, das eine kürzere Sequenz der NS3-Region und den gleichen Teil der NS4-Region enthält und ebenfalls, an Teile der SOD gekoppelt, in S. cerevisiae exprimiert wird; und Teile des NS5-Proteins, gebunden an die SOD und in S. cerevisiae exprimiert. Dieser Test wurde gemäß den Anweisungen des Herstellers ausgeführt. Seren wurden als reaktiv angesehen, wenn das entstandene Fluoreszenzsignal gleich oder größer als der Grenzwert war (sample to cutoff ratio $(S / C O) \geq 1,0$ ). Lag der $S / C O-W e r t$ im Grauzonenbereich zwischen 0,80 und 0,99, so wurde die Reaktivität als grenzwertig eingestuft und der MEIA wurde wiederholt.

Bestätigungstest (UKE SIA). Als Bestätigungstest haben wir in unserem Haus einen Immunoblot etabliert, der 4 rekombinante, in E. coli exprimierte Proteine aus den Regionen NS5, NS4, NS3 und Core des Hepatitis-C-Virus enthält. Die Größe dieser Proteine und deren Lage im HCV-Polyprotein differiert von den Antigenen, die im Suchtest eingesetzt werden [6]. Dieser Blot wurde nach unserem Krankenhaus UKE SIA (Universitäts-Klinikum Eppendorf strip immunoblot assay) genannt. In früheren Studien konnten wir zeigen, dass der UKE SIA spezifischere und seltener fragliche Ergebnisse zeigte als kommerziell vertriebene Bestätigungstests [5,6]. Der Test wurde gemäß unserer früher veröffentlichten Studie durchgeführt $[5,6]$. Zur Beurteilung der Reaktivität der einzelnen Banden wurden diese mit der internen IgG-Kontrolle verglichen. Sie wurden als stark positiv (++) bezeichnet, wenn ihre Intensität mindestens so stark war wie die der hoch konzentrierten IgG-Kontrolle, sie waren mittelhoch $(+)$ bei einer Intensität , die der der niedrig konzentrierten IgG-Kontrolle entsprach, und schwach reaktiv $((+))$, wenn sie schwächer waren als die der niedrig konzentrierten IgG-Kontrolle. Der Immunoblot war positiv, wenn mindestens 2 Banden sichtbar waren, von denen mindestens eine + oder ++ war. Fanden sich nur 2 schwach positive Banden oder nur eine Bande, so wurde das Ergebnis als fraglich bezeichnet.

Polymerase-Kettenreaktion (PCR). Die PCR wurde wie früher veröffentlicht durchgeführt [7]. Für die LightCycler ${ }^{\circledR}$-PCR (RocheBoehringer Mannheim, Mannheim) wurden $2 \mu \mathrm{l}$ der aus der RNA hergestellten cDNA, jeweils $1 \mu$ der Primer und $2 \mu$ l Mastermix, bestehend aus Deoxynukleotid-Triphosphaten, LightCycler ${ }^{\circledR}$ DNA sybr Green ${ }^{\circledR}$ und Taq-Enzym in einer Kapillare zusammen pipettiert. Mit 5 Standardkonzentrationen an HCV RNA $\left(1 \times 10^{2}\right.$, $1 \times 10^{3}, 1 \times 10^{4}, 1 \times 10^{5}$ und $1 \times 10^{6}$ Genomäquivalente $/ \mathrm{ml}$ ) sowie 2 Negativkontrollen wurde ebenso verfahren. Die Amplifikation erfolgt im LightCycler. Sybr Green ${ }^{\circledR}$ bindet an doppelsträngige DNA und führt zur Emission eines Fluoreszenzsignals, das proportional zur Menge der DNA ist. Durch Vergleich des Fluoreszenzsignals der Serumprobe mit dem der Standards erfolgt die Quantifizierung. Die Spezifität des Amplifikationsproduktes wird durch Bestimmung der Schmelztemperatur der gebildeten DNA bestätigt. Seren werden als positiv angesehen, wenn sie ein positives PCR-Signal in einem Ansatz hatten, in dem die Negativkontrollen klar negativ und die Positivkontrollen positiv waren.

Der Genotyp des HCV wurde serologisch oder durch Nukleotidsequenzierung der NS5-Region bestimmt [8]. 
Ergebnisse

Von den 1000 getesteten Patienten waren 44 im MEIA negativ, 6 hatten eine grenzwertige Reaktivität, und 950 waren reaktiv.

Patienten mit negativem MEIA-Ergebnis. 40 der 44 Patienten, die im MEIA nicht reaktiv waren, waren auch im Blot und in der PCR negativ und wurden somit als nicht infiziert bestätigt. Bei 2 weiteren Patienten war der Bestätigungstest ebenfalls negativ, aber die PCR war klar positiv mit $1 \times 10^{6} \mathrm{Kopien} / \mathrm{ml}$ bzw. $2 \times 10^{7} \mathrm{Ko}-$ pien $/ \mathrm{ml}$. In dem einen Fall handelte es sich um eine 61 Jahre alte Patientin aus Russland, die an einem myelodysplastischen Syndrom erkrankt war. Sie hatte in der letzten Zeit keine Blutprodukte erhalten und es fanden sich auch keine anderen Risikofaktoren für eine HCV-Infektion. Sie hatte jedoch eine bioptisch bestätigte Leberzirrhose. Es fanden sich keine Marker einer Hepatitis-B-Virusinfektion oder einer primär biliären Zirrhose. Die HCV-Infektion wurde in einer 2 Wochen später entnommenen Serumprobe bestätigt, in der erneut keine HCV-Antikörper gefunden wurden, aber die PCR wiederum mit $5 \times 10^{5} \mathrm{Kopien} / \mathrm{ml}$ deutlich positiv war. Durch Sequenzanalyse des PCR-Amplifikates wurde der Genotyp 1b nachgewiesen. Der andere Patient war ein 25-jähriger Medizinstudent, der im September 1998 erstmals intravenös Drogen konsumiert hatte. Im Dezember 1998 waren beide Antikörpertests negativ, aber die PCR zeigte mit $1 \times 10^{7}$ Kopien/ml eine hohe Virämie. In einer 12 Tage später entnommenen Serumprobe fanden sich noch immer keine HCVAntikörper, aber die PCR war mit $2 \times 10^{7} \mathrm{Kopien} / \mathrm{ml}$ erneut hoch positiv. Vier Wochen später war der UKE SIA noch negativ, aber der MEIA war mit einem S/CO-Wert von 3,9 erstmals schwach positiv bei weiterhin positiver PCR $\left(1 \times 10^{6} \mathrm{Kopien} / \mathrm{ml}\right)$. Somit wurde eine frische HCV-Infektion bestätigt. Durch Sequenzierung konnte der Genotyp 3a nachgewiesen werden.
Zwei weitere Seren mit negativem MEIA-Ergebnis waren im Bestätigungstest positiv, aber in der PCR konnte keine HCV-RNA nachgewiesen werden. Da keine weiteren Serumproben zur Verfügung standen, konnte die Spezifität der Reaktivität des UKE SIA nicht bestätigt werden.

Patienten mit grenzwertiger MEIA-Reaktivität. Keiner dieser 6 Patienten zeigte eine Reaktivität im Bestätigungstest oder in der PCR, so dass kein Anhalt bestand für eine HCV-Infektion.

Patienten mit positiver MEIA-Reaktivität. Insgesamt hatten 950 Patienten einen S/CO-Wert von $\geq 1,0$ im Suchtest. Tab. 1 zeigt, wie sich die S/CO-Werte dieser Patienten verteilten. Das positive Ergebnis des Suchtests wurde in 824 Fällen (86,7\%) bestätigt: in 12 Fällen durch die positive PCR, in 275 Fällen durch einen positiven Bestätigungstest, und in 537 Fällen waren sowohl der Blot als auch die PCR positiv. Von 695 dieser Patienten waren weitere Serumproben über einen Zeitraum von einem Monat bis zu 16 Jahren vorhanden; die HCV-Infektion konnte in allen Fällen bestätigt werden.

Bei 20 Patienten $(2,1 \%)$ war die PCR negativ und der Blot mit einer Bande fraglich reaktiv, so dass eine endgültige Einstufung in „infiziert“ oder „nicht infiziert“ basierend auf der Untersuchung dieser Serumprobe nicht möglich war. Bei 2 Patienten konnte die HCV-Infektion durch ein positives Ergebnis in der PCR bzw. im Bestätigungstest in einer früher untersuchten Serumprobe bestätigt werden; bei einem dieser Patienten war die PCR unter der laufenden Therapie mit Interferon negativ geworden. Bei den anderen 18 Patienten war eine abschließende Beurteilung nicht möglich, da keine weiteren Serumproben vorhanden waren.

Bei den verbleibenden 106 Patienten (11,2\%) konnte die MEIAReaktivität weder im Blot noch in der PCR bestätigt werden. Zwei davon hatten nur niedrige S/CO-Werte von unter 10; in Se-

Tab. 1: Ergebnisse in PCR und UKE SIA bei Seren, die im Suchtest (MEIA) reaktiv waren (\%).

\begin{tabular}{|c|c|c|c|c|c|c|c|}
\hline $\begin{array}{l}\text { MEIA } \\
(S / C O)\end{array}$ & $n$ & $\begin{array}{l}\text { PCR positiv } \\
\text { Blot positiv }\end{array}$ & Blot fraglich & Blot negativ & $\begin{array}{l}\text { PCR negativ } \\
\text { Blot positiv }\end{array}$ & Blot fraglich & Blot negativ \\
\hline $1-10$ & 133 & $7(5,3)$ & & & $9(6,8)$ & $14(10,5)^{1}$ & $103(77,4)^{2}$ \\
\hline $11-20$ & 42 & $7(16,7)$ & $1(2,4)$ & & $28(66,7)$ & $4(9,5)^{3}$ & $2(4,8)^{4}$ \\
\hline $21-30$ & 41 & $17(41,5)$ & $1(2,4)$ & $1(2,4)$ & $22(53,7)$ & & \\
\hline $31-40$ & 50 & $29(58,0)$ & $1(2,0)$ & & $20(40,0)$ & & \\
\hline $41-50$ & 61 & $34(55,7)$ & $4(6,6)$ & & $22(36,1)$ & & $1(1,6)$ \\
\hline $51-60$ & 71 & $47(66,2)$ & $2(2,8)$ & & $22(31,0)$ & & \\
\hline $61-70$ & 106 & $68(64,2)$ & $1(0,9)$ & & $36(34,0)$ & $1(0,9)$ & \\
\hline $71-80$ & 112 & $82(73,2)$ & & & $29(25,9)$ & $1(0,9)$ & \\
\hline $81-90$ & 123 & $85(69,1)$ & & & $38(30,9)$ & & \\
\hline $91-100$ & 110 & $85(77,3)$ & $1(0,9)$ & & $24(21,8)$ & & \\
\hline$>100$ & 101 & $76(75,2)$ & & & $25(24,8)$ & & \\
\hline Summe & 950 & $537(56,5)$ & $11(1,2)$ & $1(0,1)$ & $275(28,9)$ & $20(2,1)$ & $106(11,2)$ \\
\hline
\end{tabular}

${ }^{1}$ Ein Patient dieser Gruppe hatte vor Beginn der Therapie mit Interferon ein positives Ergebnis in der PCR und gilt somit als HCV-infiziert.

${ }^{2}$ Bei zweien dieser Patienten wurde die HCV-Infektion zu einem früheren Zeitpunkt durch ein positives PCR-Ergebnis bestätigt; bei ihnen kam es vermutlich zu einer Ausheilung.

${ }^{3}$ Bei einer Patientin dieser Gruppe wurde die HCV-Infektion vorher durch ein positives Ergebnis (3 Banden) im Blot bestätigt.

${ }^{4}$ Ein Patient dieser Gruppe hatte vor Beginn der Therapie mit Interferon ein positives Ergebnis in Blot und PCR und gilt daher als HCV-infiziert. 
ren, die ein bzw. 3 Jahre vorher untersucht worden waren, war die damals hohe Reaktivität des Suchtests sowohl im Bestätigungstest als auch durch die PCR bestätigt worden. Der Rückgang der Antikörperreaktivität in beiden Tests und die nachfolgend negative PCR lassen eine Ausheilung der HCV-Infektion vermuten mit noch verbleibender Restreaktivität im MEIA. Bei einem weiteren, ehemals drogenabhängigen Patienten mit einem S/CO-Wert von 12 waren PCR und Blot vor der 4 Jahre zuvor begonnenen Interferontherapie positiv gewesen, so dass auch dieser Patient als spezifisch reaktiv im MEIA anzusehen ist.

Somit fanden sich insgesamt 829 (87,3\%) der MEIA-Reaktivitäten bei Patienten mit aktiver oder früherer HCV-Infektion (Tab. 2). Insgesamt 103 (10,8\%) der Patienten erwiesen sich als falsch reaktiv. Von 43 dieser Patienten waren weitere Serumproben über einen Zeitraum von einem Monat bis zu 8 Jahren vorhanden; 3 davon waren nur vorübergehend im Suchtest reaktiv, bei den anderen 40 blieb die in keinem Test zu bestätigende Reaktivität des Suchtests erhalten. Eine endgültige Klassifizierung war bei 18 Patienten (1,9\%) nicht möglich. Die Häufigkeit, mit der falsch positive Reaktivitäten auftraten, hing deutlich von der Höhe der S/CO-Werte ab: während 75,9\% der Reaktivitäten im Bereich zwischen 1 und 10 sich als falsch erwiesen, konnte nur noch eine der Reaktivitäten $(0,1 \%)$ mit einem Wert von über 40 nicht bestätigt werden (Tab. 2). Auch die Anzahl der fraglichen Ergebnisse variierte mit dem S/CO-Wert: 9,8\% der Reaktivitäten unter 10 konnten nicht endgültig beurteilt werden; bei den Werten über 10 waren es nur noch 0,6\% (Tab. 2).

\section{Diskussion}

Die Entwicklung der ersten Antikörpertests zur Detektion von HCV-Antikörpern war ein wesentlicher Schritt in der Diagnostik der meisten der früher im Formenkreis der Hepatitis Non-A, Non-B zusammengefassten Hepatitiden; aber es zeigte sich auch, dass sowohl die Suchtests, die nur das c100 - 3 der NS4-Region enthalten, als auch die Bestätigungstests der 1. Generation eine niedrige Sensitivität und eine hohe Rate an unspezifischen Reaktivitäten aufwiesen [9]. Durch den Einschluss weiterer Anti-

Tab. 2: Klassifizierung der Seren mit MEIA-Reaktivität unter Berücksichtigung aller Ergebnisse (\%).

\begin{tabular}{lrrrrrrr}
\hline $\begin{array}{l}\text { MEIA } \\
\text { (S/CO) }\end{array}$ & \multicolumn{3}{l}{ spezifischreaktiv } & \multicolumn{1}{l}{ fraglich } & & \multicolumn{2}{l}{ HCV negativ } \\
\hline $1-10$ & 133 & 19 & $(14,3)$ & 13 & $(9,8)$ & 101 & $(75,9)$ \\
\hline $11-20$ & 42 & 38 & $(90,5)$ & 3 & $(7,1)$ & 1 & $(2,4)$ \\
\hline $21-30$ & 41 & 41 & $(100,0)$ & & & & \\
\hline $31-40$ & 50 & 50 & $(100,0)$ & & & \\
\hline $41-50$ & 61 & 60 & $(98,4)$ & & 1 & $(1,6)$ \\
\hline $51-60$ & 71 & 71 & $(100,0)$ & & & \\
$61-70$ & 106 & 105 & $(99,1)$ & 1 & $(0,9)$ & & \\
$71-80$ & 112 & 111 & $(99,1)$ & 1 & $(0,9)$ & & \\
$81-90$ & 123 & 123 & $(100,0)$ & & & & \\
\hline $91-100$ & 110 & 110 & $(100,0)$ & & & & \\
\hline$>100$ & 101 & 101 & $(100,0)$ & & & & \\
\hline Summe & 950 & 829 & $(87,3)$ & 18 & $(1,9)$ & 103 & $(10,8)$ \\
\hline
\end{tabular}

gene (c33c aus der NS3-Region, c22-3 aus der Core-Region) in den Such- und Bestätigungstests der 2. Generation besserten sich Sensitivität und Spezifität $[3,10]$. Durch die Hinzunahme eines weiteren Antigens aus dem nicht-strukturellen Bereich NS5 sollte die Sensitivität weiter gesteigert werden. Die in den ersten Studien mit diesen Tests beschriebene höhere Sensitivität und Spezifität war jedoch auf die Modifikation des c33-Proteins zurückzuführen [11,12]. Unsere Studie belegt, dass die kommerziellen Screening-Tests der 3. Generation alleine für eine verlässliche Diagnose einer HCV-Infektion nicht geeignet sind. Die Sensitivität erwies sich mit 2 von 50 falsch negativen Seren als zufriedenstellend, aber die Spezifität ist mit 10,8\% falsch reaktiven Seren viel zu niedrig. In einem Blutspenderkollektiv, in dem der positive Vorhersagewert eines positiven HCV- Screening-Assays ohnehin niedrig ist [13], führt eine falsch positive Reaktivität zum Ausschluss eines ansonsten gesunden Spenders und somit zu einer geringeren Verfügbarkeit von Blutspenden. Aber auch bei Patienten mit klinischen Zeichen einer Hepatitis kann eine schwache Antikörperreaktivität, die in keinem Bestätigungstest auf die Spezifität hin untersucht wurde, zu einer falschen Diagnose einer HCV-Infektion führen. Dadurch kann eine nicht gerechtfertigte Kombinationstherapie mit Interferon und Ribavirin erfolgen, die nicht nur sehr teuer ist, sondern auch zahlreiche Nebenwirkungen hat. Außerdem kann die wahre Ursache der Hepatitis übersehen werden.

Wir fanden eine deutliche Assoziation zwischen der Höhe der Reaktivität und der Bestätigung im UKE SIA oder in der PCR. Ergebnisse mit einem S/CO-Wert $<10$ lassen sich nur selten bestätigen, während solche $>40$ sich fast immer als spezifisch erweisen. Insgesamt war eine endgültige Diagnose bei 18 (1,9\%) der Patienten nicht möglich, da sie nur eine Bande im Blot zeigten und die PCR negativ war. Für eine solche fragliche Reaktivität im Such- oder Bestätigungstest gibt es verschiedene Erklärungen. Werden rekombinante Testantigene benutzt, so können Antikörper gegen den zur Klonierung und Expression benutzten Mikroorganismus zu einer unspezifischen Reaktivität führen [14]. So wurde bei Tests, die synthetische Peptide verwenden, in früheren Studien eine höhere Spezifität beschrieben, aber sie erwiesen sich leider auch als weniger sensitiv $[14,15]$. Ein hoher IgGSpiegel im Serum kann zu einer unspezifischen Bindung der Fc-Fragmente an die feste Phase und so zu einem falsch positiven Ergebnis im Screening-Test führen. So wurden hohe Raten unspezifischer Reaktivitäten bei Patienten mit rheumatischer Arthritis, Sjögren-Syndrom, Autoimmunhepatitis, primär-biliärer Zirrhose oder Kryoglobulinämie beschrieben [16]. Auch nach Ausheilung einer HCV-Infektion kann als einziger Marker eine schwache Reaktivität im Suchtest oder eine einzelne Bande im Bestätigungstest verbleiben; wir vermuten, dass dies für 2 unserer Patienten zutrifft, bei denen zu einem früheren Zeitpunkt ein positives Ergebnis in der PCR und im Blot gefunden worden war und die nun nur noch schwach reaktiv im MEIA waren bei negativen Befunden in Blot und PCR. Die spontane Ausheilung einer Hepatitis C ist aber ein seltenes Ereignis [17] und kann somit nicht die Mehrzahl der niedrigen Reaktivitäten erklären. Unter der Therapie mit Interferon und Ribavirin kann ebenfalls die Antikörperreaktivität absinken; dies war bei 2 weiteren Patienten der Fall. Bei den meisten Patienten findet sich jedoch keine Erklärung für die schwache Reaktivität in Such- oder Bestätigungstests; unsere Studie zeigt, dass diese schwachen Ergebnisse 
meist über einen langen Zeitraum von bis zu 8 Jahren persistieren.

Im Rahmen einer Immundefizienz, wie z.B. bei niereninsuffizienten Patienten unter chronischer Hämodialyse, kann bei einer bestehenden HCV-Infektion die Antikörperbildung schwach sein oder fehlen [18]. In unserer Studie hatten 11 von 31 Patienten (35,5\%) mit nur einer Blotbande ein positives PCR-Ergebnis. Wir haben keinen Hinweis darauf, dass eine zugrunde liegende Immunsuppression die Ursache der schwachen Immunantwort bei den restlichen 20 Patienten mit negativem PCR-Ergebnis war. Seren von Patienten mit schwachen oder fraglichen Antikörpertests sowie von immunsupprimierten Patienten sollten in jedem Fall mit der PCR untersucht werden.

Wie kann die HCV-Diagnostik verbessert werden? Werden Reaktivitäten des Suchtests mit kommerziellen Bestätigungstests nachuntersucht, so detektieren letztere häufig den gleichen unspezifischen Antikörper wie die Suchtests, da die Antigene beider Tests identisch sind. Deshalb wäre es wünschenswert, dass die Bestätigungstests immundominante Antigene enthalten, die sich klar von denen der Suchtests unterscheiden [19]. Außerdem beschränken sich die in den kommerziellen Tests verwendeten Antigene auf den in den USA dominierenden Genotyp 1a; diese Antigene entsprechen aber nicht unbedingt den Genotypen, die in anderen Teilen der Welt vorkommen $(15,16,20)$. So erwies sich in einer früher veröffentlichten Studie der in unserem Haus entwickelte Blot mit Antigenen aus unseren lokalen Virusisolaten gegenüber den kommerziellen Tests als überlegen [6]. Diese repräsentativeren Antigene führten wahrscheinlich auch dazu, dass die Anzahl der Patienten (1,9\%), bei denen eine abschließende Beurteilung nicht möglich war, gering war. Zusammenfassend hat die Einbeziehung des NS5-Antigens in die Antikörpertests der 3. Generation nicht zu einer Verbesserung der Spezifität geführt. Jedes Labor, das die Diagnostik von HCVInfektionen anbietet, sollte seinen Screening-Assay sorgfältig evaluieren und Breakpoints etablieren, bis zu denen Reaktivitäten des Suchtests meist unspezifisch sind bzw. bei deren Überschreiten eine Bestätigung in weiteren Tests zu erwarten ist. Im Rahmen der HCV-Diagnostik dient die PCR nicht nur zur Bestimmung der Viruslast und somit der Infektiosität, sondern hat mehr den Charakter eines Bestätigungstests. Jedoch auch bei chronischer HCV-Infektion gibt es Phasen der niedrigen Virämie, so dass ein negatives PCR-Ergebnis in einer Serumprobe eine bestehende HCV-Infektion nicht ausschließt und auch nicht die Ausheilung der Infektion belegt [17]. Die Diagnose einer HCV-Infektion kann nicht allein auf der Basis eines positiven Suchtests gestellt werden; vielmehr bedarf dies immer einer weiterführenden Diagnostik mit einem Bestätigungstest, der seinen Namen eigentlich nur verdient, wenn er Antigene enthält, die sich in Herstellung und Herkunft von denen des Suchtests unterscheiden.
Literatur

${ }^{1}$ Choo QL, Kuo G, Weiner AJ, Overby LR, Bradley DW, Houghton M. Isolation of a c-DNA clone derived from a blood borne non-A, non-B viral hepatitis genome. Science 1989; 244: 359-362

2 Kuo G, Choo QL, Alter HJ, Gitnick GL, Redeker AG, Purcell AH, Miyamura T, Dienstag JL, Alter MJ, Stevens CE, Tegtmeier GE, Bonino F, Colombo M, Lee WS, Kuo C, Berger K, Shuster JR, Overby LR, Bradley DW, Houghton M. An assay for circulating antibodies to a major etiologic virus of human non-A, non-B hepatitis. Science 1989; 244: $362-364$

${ }^{3}$ Alter H. New kit on the block: evaluation of second-generation assays for detection of antibody to the hepatitis C virus. Hepatology 1992; 15: $350-353$

${ }^{4}$ Zaaijer HL, Vallari DS, Cunnigham M, Lesniewski R, Reesink HW, van der Poel CL, Lelie PN. E2 and NS5: new antigens for detection of hepatitis C virus antibodies. J. Med. Virol 1994; 44: 395- 397

${ }^{5}$ Schröter M, Feucht HH, Schäfer P, Zöllner B, Polywka S, Laufs R. Definition of false-positive reactions in screening for hepatitis $C$ virus antibodies. J. Clin. Microbiol 1999; 37: 233-234

${ }^{6}$ Feucht HH, Zöllner B, Polywka S, Laufs R. Study on reliability of commercially available hepatitis $C$ virus antibody tests. J. Clin. Microbiol 1995; 33: 620-624

${ }^{7}$ Schröter M, Zöllner B, Schäfer P, Laufs R, Feucht HH. Quantitative detection of hepatitis $C$ virus RNA by light cycler PCR and comparison with two different PCR assays. J. Clin. Virol 2001; 39: 765-768

${ }^{8}$ Schröter M, Feucht HH, Schäfer P, Zöllner B, Laufs R. Serological determination of hepatitis $C$ virus subtypes $1 \mathrm{a}, 1 \mathrm{~b}, 2 \mathrm{a}, 2 \mathrm{~b}, 3 \mathrm{a}$, and $4 \mathrm{a}$ by a recombinant immunoblot assay. J. Clin. Microbiol 1999; 37: $2576-2580$

${ }^{9}$ Chaudhary RK, Frenette S, Mo T. Evaluation of hepatitis C virus kits. J. Clin. Microbiol 1991; 29: 2616-2617

${ }^{10}$ Katayama T, Mazda T, Kikuchi S, Harada S, Matsuura Y, Chiba J, Ohba H, Saito I, Miyamura T. Improved serodiagnosis of Non-A, Non-B hepatitis by an assay detecting antibody to hepatitis $C$ virus core antigen. Hepatology 1992; 15: $391-394$

${ }^{11}$ Damen M, Zaaijer HL, Cuypers HTM, Vrielink H, van der Poel CL, Reesink HW, Lelie PN. Reliability of the third-generation recombinant immunoblot assay for hepatitis $C$ virus. Transfusion 1995; 35: 745 - 749

12 Lee SR, Wood CL, Lane MJ, Francis B, Gust C, Higgs CM, Nelles MJ, Polito A, DiNello R, Achord D. Increased detection of hepatitis C virus infection in commercial plasma donors by a third-generation screening assay. Transfusion 1995; 35: 845 - 849

${ }^{13}$ Bresters D, Zaaijer HL, Cuypers HTM, Reesink HW, Winkel IN, van ExelOehlers PJ, van Drimmelen AAJ, Jansen PLM, van der Poel CL, Lelie PN. Recombinant immunoblot assay reaction patterns and hepatitis $C$ virus RNA in blood donors and non- $A$, non-B hepatitis patients. Transfusion 1993; 33: 634-638

${ }^{14}$ Léon P, López JA, Echevarría JM. Evaluation of laboratory assays for screening antibody to hepatitis C virus. Transfusion 1993; 33: $268-270$

${ }^{15}$ Callahan JD, Constantine NT, Kataaha P, Zhang X, Hyams KC, Bansal J. Second generation hepatitis $C$ virus assays: performance when testing African sera. J. Med. Virol 1993; 41: 35-38

${ }^{16}$ Gross JB, Persing DH. Hepatitis C: advance in diagnosis. Mayo Clin. Proc 1995; 70: $296-297$

${ }^{17}$ Feucht HH, Zöllner B, Schröter M, Polywka S, Buggisch P, Nolte H, Laufs R. High rate of chronicity in HCV infection determined by antibody confirmatory assays and PCR in 4110 patients during long-term follow-up. J. Clin. Virol 1999; 13: 43-51

18 Bukh J, Wantzin P, Krogsgaard K, Knudsen F, Purcell RH, Miller RH and the Copenhagen Dialysis HCV Study Group. High prevalence of hepatitis $C$ virus RNA in dialysis patients: failure of commercially available tests to identify a significant number of patients with HCV infection. J. Infect. Dis 1993; 168: $1343-1348$

${ }^{19}$ Nübling CM, von Wangenheim G, Staszewski S, Löwer J. Hepatitis C virus antibody among immunodeficiency virus-1-infected individuals: analysis with different test systems. J. Med. Virol 1994; 44: 49-53

${ }^{20}$ Dhaliwal SK, Prescott LE, Dow BC, Davidson F, Brown H, Yap PL, Follett EAC, Simmonds P. Influence of viraemia and genotype upon serological reactivity in screening assays for antibody to hepatitis $\mathrm{C}$ virus. J. Med. Virol 1996; 48: 184-190 